\title{
Evolução da migração de partos para Aracaju, Sergipe, Brasil, 1970-1996
}

\author{
Shift in demand for childbirth services \\ from rural Sergipe State to the capital city, \\ Aracaju, Brazil, 1970-1996
}

Ricardo Queiroz Gurgel 1

Vera Lúcia Alves França ${ }^{2}$

Diana Melo de Matos 3

\footnotetext{
1 Núcleo de Pós-Graduação em Medicina, Universidade Federal de Sergipe.

Rua Cláudio Batista s/n, Aracaju, SE 49060-100, Brasil. ricardoqg@infonet.com.br

2 Núcleo de Pós-Graduação em Geografia, Universidade Federal de Sergipe. Campus Universitário Prof. José Aloísio de Campos, Bloco de Didática II, São Cristóvão, SE 49100-000, Brasil. soninha@infonet.com.br 3 Hospital Universitário, Universidade Federal de Sergipe. Rua Cláudio Batista s/n, Aracaju, SE 49060-100, Brasil. diana.matos@bol.com.br
}

\begin{abstract}
This study analyzes trends in the migration of childbirth from rural areas of Sergipe State, Brazil, to the capital city, Aracaju, from 1970 to 1996. Data on "mother's place of residence" were obtained from mothers whose children were born in maternity hospitals in Aracaju in 1970, 1976, 1986, and 1996. Significant differences occurred in the proportion of mothers who resided outside of Aracaju but came there to give birth, especially from 1976 to 1986. This shift was stimulated by improvement of State highways and the granting of personal political favors in exchange for votes. Changes in the State hospital structure did not appear to have influenced the childbirth shift to Aracaju. There was a $134.6 \%$ increase in deliveries occurring in the State capital. There were also different trends among the various regions of the State, with some regional health districts displaying a downward trend in the number of deliveries from 1986 to 1996. It is urgent that more regional and hierarchical support be provided for childbirth and neonatal care in order to ensure quality assistance for pregnant women and their infants.
\end{abstract}

Key words Delivery; Perinatal Care; Maternity Hospitals

Resumo O objetivo deste estudo é descrever a evolução da migração de partos do interior do Estado de Sergipe para a capital (Aracaju), no período compreendido entre 1970 e 1996. Para tanto utilizou-se a informação "município de residência da mãe" cujo parto ocorreu nas maternidades de Aracaju, nos anos de 1970, 1976, 1986 e 1996. Ao se estudar as proporções de mães não residentes em Aracaju, verificaram-se diferenças significativas, sendo que o período com maiores percentuais de migração ocorreu entre 1976 e 1986. A migração foi estimulada pela melhoria das rodovias que dão acesso à capital (a partir de 1970) e pelo incentivo político com fins eleitoreiros (clientelismo). Assim, a melhoria na estrutura hospitalar do interior não impediu o aumento da migração para a capital. Em Sergipe, no período em estudo, houve um crescimento de 134,6\% do número de partos ocorridos na capital, provenientes de outras localidades. Além disso, observase intensidades de variação diferenciadas, quando se analisa o fenômeno por regiões, tendo em vista a melhoria das condições de acessibilidade. Faz-se necessária a regionalização e hierarquização da assistência ao parto e ao recém-nascido, para que se possa dar um atendimento adequado às gestantes e aos seus filhos.

Palavras-chave Parto; Assistência Perinatal; Maternidades 


\section{Introdução}

Os serviços de saúde no Brasil concentram-se nas capitais, nas áreas urbanas e nas zonas centrais, em detrimento das zonas rurais, das áreas mais pobres e periféricas, determinando, junto com outros fatores socioeconômicos e culturais, uma extrema desigualdade na distribuição e oferta dos serviços de saúde, tendo como conseqüência uma maior dificuldade no acesso aos mesmos (Buss, 1993; Campos \& Carvalho, 2000; Victora et al., 1994).

$\mathrm{O}$ acesso dificultado aos serviços de saúde gera falhas na assistência médica, como demonstrado por diversos estudos, sendo um dos mais clássicos o Black Report (Townsend \& Davidson, 1982), que mostrou a desigualdade na assistência médica na Inglaterra, além da desigualdade no adoecer e morrer. Na assistência perinatal, Ribeiro \& Silva (2000), em São Luís e Gomes \& Santo (1997), em São Paulo, encontraram elevados índices de mortalidade neonatal, por causas que seriam evitáveis através de um melhor acompanhamento do trabalho de parto e assistência pediátrica no pós-parto imediato. Leal \& Szwarcwald (1996) verificaram no Rio de Janeiro, elevado número de óbitos ocorridos na primeira hora após o nascimento, causados por esses mesmos motivos.

A desigualdade no acesso fica expressa na distribuição espacial da ocorrência de eventos indesejáveis de saúde, como é o caso das mortes neonatais, com um componente social refletido nessas diferenças (Gouveia et al., 1987; Morais Neto et al., 2001; Shimakura et al., 2001). Reduzir as desigualdades de oferta e acesso aos serviços de saúde nos países em desenvolvimento, é meta prioritária do plano Saúde para todos no Ano 2000, da Organização Mundial da Saúde, desde 1978 (Silva et al., 2000).

O presente estudo tem por objetivo descrever e mapear, para o período de 1970 a 1996, o deslocamento das gestantes do seu município de residência, no interior do Estado de Sergipe, para a capital Aracaju, por ocasião do parto, em busca de assistência obstétrica, considerando a relação com a oferta de serviços de saúde.

\section{Metodologia}

O universo de análise é a ocorrência de nascimentos no Município de Aracaju em 1970, 1976, 1986 e 1996. Os dados foram coletados em quatro maternidades da cidade, considerando a informação "município de residência da mãe" nesses anos. A coleta foi feita no livro de registro de procedimento ou nos prontuários indi- viduais, quando aquele não estava disponível. Aracaju tinha $98 \%$ dos partos ocorrendo em ambiente hospitalar durante o período estudado. Em uma das maternidades da cidade, a perda total do arquivo impediu a coleta, resultando assim, na exclusão desses nascimentos, o que corresponde a cerca de $25 \%$ do total. A clientela dessa maternidade pouco variou no decorrer do tempo mantendo o predomínio de gestantes provenientes do SUS (Sistema Único de Saúde), além do atendimento em menor número de outros convênios e particulares. Assim, foi considerado que os $75 \%$ de nascimentos estudados foi representativo para a explicação do fenômeno.

Os dados foram agrupados em residentes e não residentes em Aracaju, e por Distritos Regionais de Saúde (DRS) do município de residência da mãe, em valores absolutos e relativos, para os anos 1970, 1976, 1986 e 1996 (Tabelas 1 e 2), e por variação relativa da migração nos diversos períodos (Tabela 3). Os 75 municípios do Estado de Sergipe estão agrupados nos 7 DRS pela Secretaria de Estado da Saúde, com a finalidade de facilitar o gerenciamento e implementação das políticas de saúde. A distribuição dos municípios por DRS considera a proximidade geográfica, a facilidade de acesso, o desenvolvimento econômico e o fluxo natural já existente entre os municípios. As sedes dos DRS são: Estância no DRS I, Itabaiana no DRS II, Maruim no III, Porto da Folha no IV, Propriá no V, Lagarto no VI e Aracaju no DRS VII (Secretaria de Estado da Saúde de Sergipe, 1987).

Ainda foram coletados dados sobre a estrutura hospitalar no estado (CONDESE, 1971; SEPLAN, 1987; SEPLANTEC, 1997), o número de ambulâncias (DETRAN-SE, 2001) e junto ao Departamento de Estradas de Rodagens (DER-SE, 1979, 1982, 1987) dados referentes à cronologia de abertura e pavimentação das rodovias.

Posteriormente, foram elaborados cartogramas de fluxo dos partos do município de residência da mãe em direção a Aracaju, local onde se deu o parto, através das principais estradas que fazem acesso à capital. A largura de cada linha corresponde ao número de gestantes que migraram, e as linhas vão se somando até totalizarem o número de partos de não residentes em Aracaju em cada ano. As informações foram mapeadas considerando os fluxos do local de residência até o do parto.

Além disso, foram elaborados através do Sistema de Informações Geográficas Atlas GIS (França \& Torres, 1998), cartogramas referentes à estrutura hospitalar do Estado. 
Partos ocorridos nas maternidades de Aracaju, Sergipe, Brasil, 1970-1996.

\begin{tabular}{|c|c|c|c|c|c|c|c|c|}
\hline & \multicolumn{2}{|c|}{1970} & \multicolumn{2}{|c|}{1976} & \multicolumn{2}{|c|}{1986} & \multicolumn{2}{|c|}{1996} \\
\hline & $\mathrm{N}$ & $\%$ & $\mathrm{~N}$ & $\%$ & $\mathrm{~N}$ & $\%$ & $\mathrm{~N}$ & $\%$ \\
\hline Residentes em Aracaju & 4.913 & 88,22 & 7.069 & 82,11 & 8.196 & 63,74 & 7.627 & 58,38 \\
\hline $\begin{array}{l}\text { Não residentes } \\
\text { em Aracaju }\end{array}$ & 656 & 11,78 & 1.540 & 17,89 & 4.662 & 36,26 & 5.438 & 41,62 \\
\hline Total & 5.569 & 100,00 & 8.609 & 100,00 & 12.858 & 100,00 & 13.065 & 100,00 \\
\hline
\end{tabular}

Fonte: Maternidades Francino Melo, Hildete Falcão Baptista, Santa Helena e Santa Lúcia.

$\chi^{2}=2.522,27 ; p<0,01$.

Partos ocorridos nas maternidades de Aracaju, Sergipe, Brasil, por Distritos Regionais de Saúde (DRS) do município de residência da mãe, 1970, 1976, 1986 e 1996.

\begin{tabular}{|c|c|c|c|c|c|c|c|c|}
\hline & \multicolumn{2}{|c|}{1970} & \multicolumn{2}{|c|}{1976} & \multicolumn{2}{|c|}{1986} & \multicolumn{2}{|c|}{1996} \\
\hline & $\mathrm{N}$ & $\%$ & $\mathrm{~N}$ & $\%$ & $\mathrm{~N}$ & $\%$ & $\mathrm{~N}$ & $\%$ \\
\hline DRS I & 11 & 0,20 & 100 & 1,16 & 459 & 3,57 & 357 & 2,73 \\
\hline DRS II & 37 & 0,66 & 87 & 1,01 & 631 & 4,91 & 245 & 1,87 \\
\hline DRS III & 102 & 1,83 & 169 & 1,96 & 640 & 4,98 & 886 & 6,78 \\
\hline DRS IV & 34 & 0,61 & 44 & 0,51 & 402 & 3,13 & 423 & 3,24 \\
\hline DRS V & 14 & 0,25 & 52 & 0,60 & 198 & 1,54 & 381 & 2,92 \\
\hline DRS VI & 43 & 0,77 & 127 & 1,48 & 541 & 4,21 & 617 & 4,72 \\
\hline DRS VII & 5.298 & 95,14 & 7.578 & 88,03 & 9.712 & 75,53 & 9.976 & 76,36 \\
\hline Outros & 17 & 0,31 & 36 & 0,42 & 133 & 1,03 & 170 & 1,30 \\
\hline Ignorado & 13 & 0,23 & 416 & 4,83 & 142 & 1,10 & 10 & 0,08 \\
\hline Total & 5.569 & 100,00 & 8.609 & 100,00 & 12.858 & 100,00 & 13.065 & 100,00 \\
\hline
\end{tabular}

Fonte: Maternidades Francino Melo, Hildete Falcão Baptista, Santa Helena e Santa Lúcia.

\section{Resultados}

Anualmente, em Sergipe, registram-se aproximadamente 40 mil nascimentos com cerca de 95\% acontecendo em ambiente hospitalar, sendo que $51 \%$ ocorrem em Aracaju. A partir de 1970, verifica-se um aumento crescente na participação de parturientes provenientes do interior do Estado de Sergipe (não residentes em Aracaju), nos partos ocorridos nas maternidades da cidade: $11,78 \%, 17,89 \%, 36,26 \%$ e $41,62 \%$, em 1970, 1976, 1986 e 1996, respectivamente (Tabela 1).

Tal aumento ocorreu em todos os DRS, no período de 1970 a 1986, exceto no DRS VII, do qual faz parte Aracaju, pois o aumento relativo dos outros DRS aconteceu em detrimento da diminuição relativa desta. No período entre 1986 a 1996, ocorreu uma tendência à estabili-
Tabela 3

Variação relativa da migração de partos para Aracaju, Sergipe, Brasil, 1970-1996.

\begin{tabular}{lrrrr}
\hline \multirow{2}{*}{ DRS } & \multicolumn{4}{c}{ Variação relativa } \\
& $1970 / 1976$ & $1976 / 1986$ & $1986 / 1996$ & $1970 / 1996$ \\
\hline DRS I & 809,09 & 359,00 & $-22,22$ & $3.145,45$ \\
DRS II & 135,13 & 625,28 & $-61,17$ & 562,16 \\
DRS III & 65,68 & 278,69 & 38,43 & 768,62 \\
DRS IV & 29,41 & 813,63 & 5,22 & $1.144,11$ \\
DRS V & 271,42 & 280,76 & 92,42 & $2.621,42$ \\
DRS VI & 195,34 & 325,98 & 14,04 & $1.334,88$ \\
DRS VII & 43,03 & 28,16 & 2,71 & 88,29 \\
Outros & 111,76 & 269,44 & 27,81 & 900,00 \\
Total & 54,58 & 49,35 & 1,60 & 134,60 \\
\hline
\end{tabular}

Fonte: Maternidades Francino Melo, Hildete Falcão Baptista, Santa Helena e Santa Lúcia. 
zação desses percentuais, com redução nos DRS I (3,57\% em 1986 e $2,73 \%$ em 1996) e DRS II $(4,91 \%$ em 1986 e $1,87 \%$ em 1996) e aumento nos demais DRS, porém em ritmo menor (Tabela 2).

O período com maior variação relativa de migração foi aquele entre 1976 e 1986, enquanto que de 1986 a 1996 ocorreu uma desaceleração dessa tendência, inclusive com valores negativos nos DRS I $(-22,22 \%)$ e DRS II $(-61,17 \%)$ (Tabela 3).

As Figuras 1 e 2, mostram o fluxo de parturientes deslocando-se dos seus municípios de residência até Aracaju, no momento do parto em 1970 e 1996. Nota-se um aumento considerável da intensidade desse fenômeno, como se pode ver pelo espessamento das linhas e o aumento do valor de referência na legenda.

Nas Figuras 3 e 4, estão representados os estabelecimentos de saúde (em geral e as maternidades) nos dois períodos (1970 e 1996). De fato, verifica-se um aumento expressivo de maternidades e hospitais, tanto em Aracaju como em outros municípios do Estado.

\section{Discussão}

A migração de gestantes do seu município de residência para outro, por ocasião do parto, parece ser comum em regiões com características de metropolização como é o caso de Aracaju. Bettiol et al. (1998) observaram que no Município de Ribeirão Preto $20,5 \%$ dos nascimentos eram procedentes de outras cidades. A comparação entre Ribeirão Preto e Aracaju é pertinente, pois as duas têm o mesmo porte e a mesma função de centros regionais submetropolitanos, no conjunto do sistema urbano brasileiro, sendo que em Aracaju o fenômeno é mais intenso, certamente em função das diferenças nas redes urbanas dos dois Estados (São Paulo e Sergipe). Sergipe apresenta uma rede urbana primaz em que Aracaju aparece comandando todo o sistema. Isso ocorre em função da exigüidade do território e da forma da malha viária, que oferece boas condições de acessibilidade.

Segundo o DER, em Sergipe houve uma melhoria e ampliação da malha rodoviária no período analisado neste estudo, passando de $165 \mathrm{~km}$ de rodovias pavimentadas em 1974 para 1.284km em 1987 (DER-SE, 1974, 1979, 1982, 1987). De fato, a melhoria da infra-estrutura das rodovias e a facilidade de maior acesso aos meios de transporte se constituem em elementos responsáveis pelo incremento da migração, ao lado de outros fatores como a presença de maternidades mais bem equipadas na capital.
Em 1970, Sergipe dispunha de 33 unidades hospitalares, sendo que apenas 13 possuíam serviços obstétricos. Em 1996, esses números se elevaram passando para 60 hospitais e 33 maternidades, sendo que a capital concentra o maior número deles. No período analisado, Aracaju passou a ter quatro maternidades, correspondendo a uma variação de $300 \%$. O número de maternidades é ampliado em 166\% e o de hospitais em $81 \%$ (Figuras 3 e 4 ).

A fragilidade da rede hospitalar no interior do Estado de Sergipe e a melhoria das principais rodovias que dão acesso à capital, facilitam a ação de políticos, que usam ambulâncias e outros veículos para conduzir as pessoas (França, 1999).

Mendonza-Sassi \& Béria (2001) mostraram com vários exemplos que a distância entre a casa e o serviço de saúde por si só não gera efeitos significativos, a não ser nos casos em que há dificuldade de acesso, como em áreas montanhosas, o que não ocorre em Sergipe.

A migração de partos para Aracaju deu um salto entre 1976 e 1986, apesar do aumento do número de hospitais e da disseminada redução das taxas de natalidade no estado (IBGE, 1970, 1991). De fato, o maior aumento foi observado entre 1970 e 1986, havendo uma tendência à estabilização, inclusive com redução nas DRS I e II no último período (1986-1996).

Segundo Campos et al. (2000), a eqüidade em saúde pode ser entendida como a igualdade de acesso à saúde e esse acesso envolve o adequado planejamento da localização dos serviços de saúde. Em Sergipe o que promoveu a migração não foi apenas a má distribuição dos hospitais no estado, mas a qualidade do atendimento e outros fatores como melhoria das rodovias, maior acesso aos meios de transporte e incentivo político à migração para a capital à procura de serviços em troca de favores eleitoreiros (clientelismo). Em 1970, existiam apenas 13 ambulâncias no estado, enquanto que em 1996, havia 126 ambulâncias registradas no DETRAN, configurando-se um aumento de $869 \%$ (DETRAN-SE, 2001).

A intensidade de migração é forte em todo o período nos municípios mais próximos da capital, sobretudo daqueles que fazem parte da DRS VII. Em 1970, as migrantes eram provenientes de 53 municípios, passando para os 74 em 1986, com acréscimos acentuados da migração (Figura 1). A intensidade de migração era menor em 1970, abrangendo municípios mais próximos, embora já chegasse ao sudoeste, ao noroeste e ao centro do Estado (Figura 2). Em 1996, os fluxos refletem a maior intensidade de deslocamentos oriundos de 74 municí- 
Figura 1

Migração de partos. Sergipe, Brasil, 1970

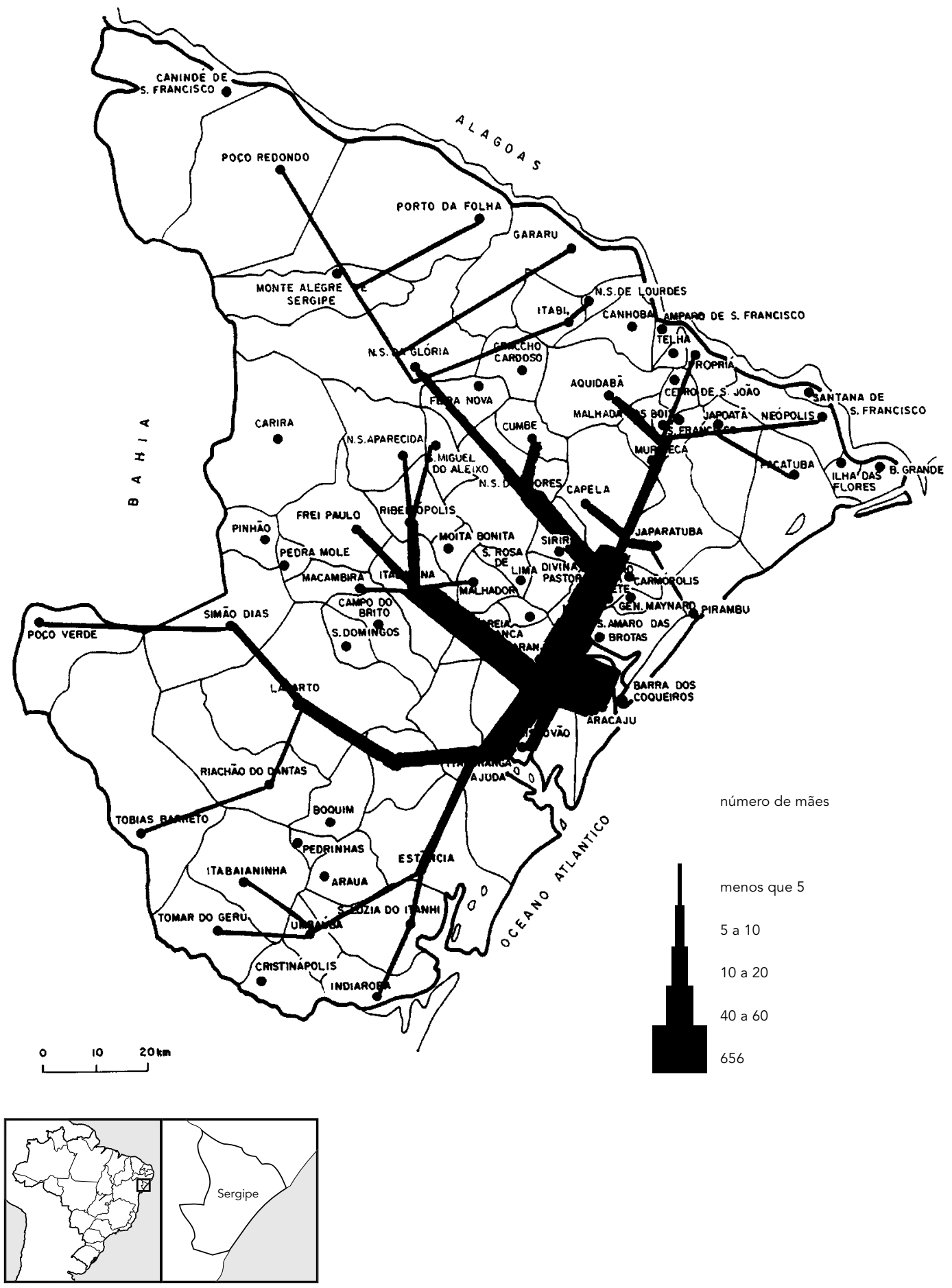


Figura 2

Migração de partos. Sergipe, Brasil, 1996.

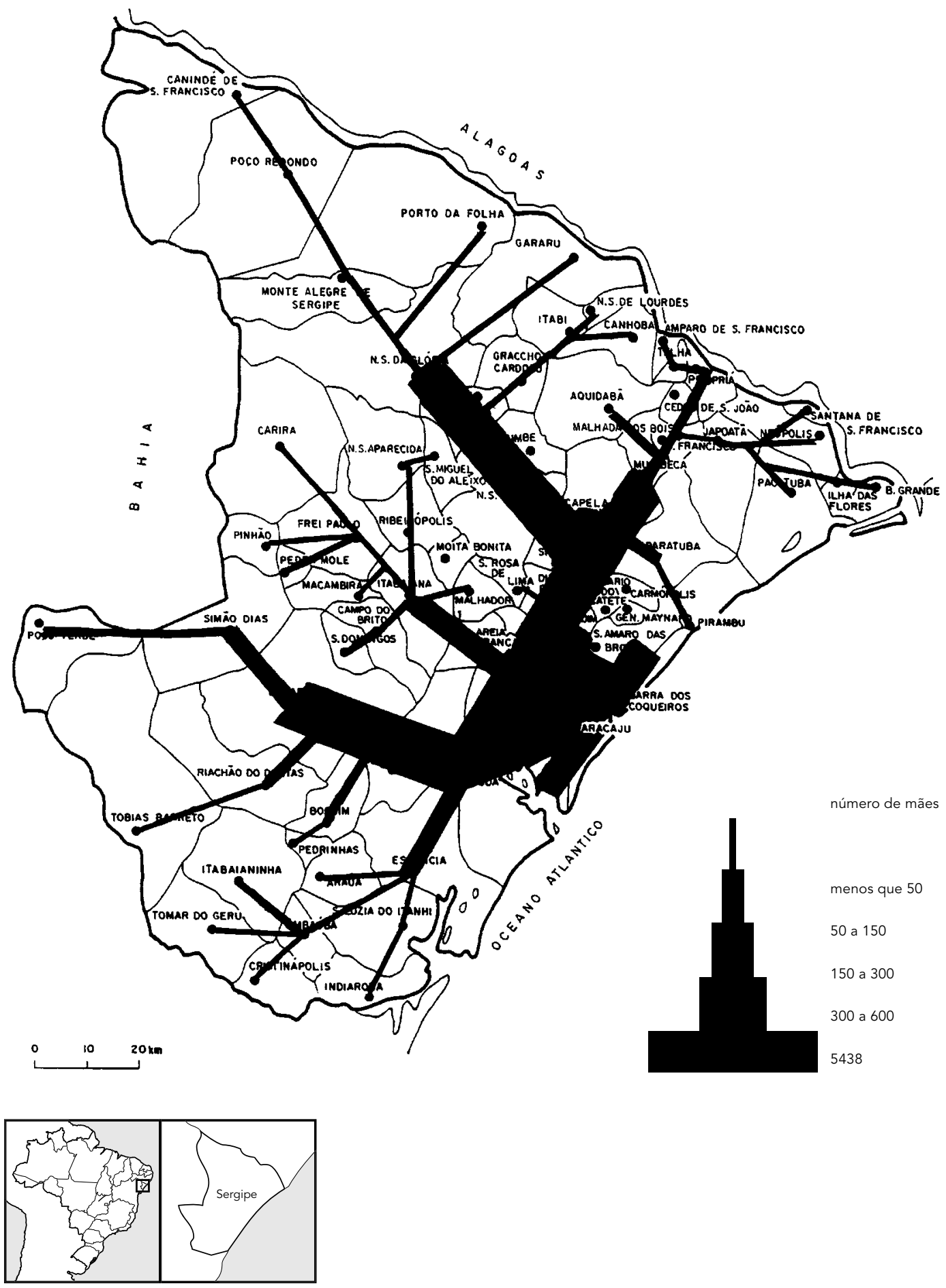


Hospitais. Sergipe, Brasil, 1970 e 1996.
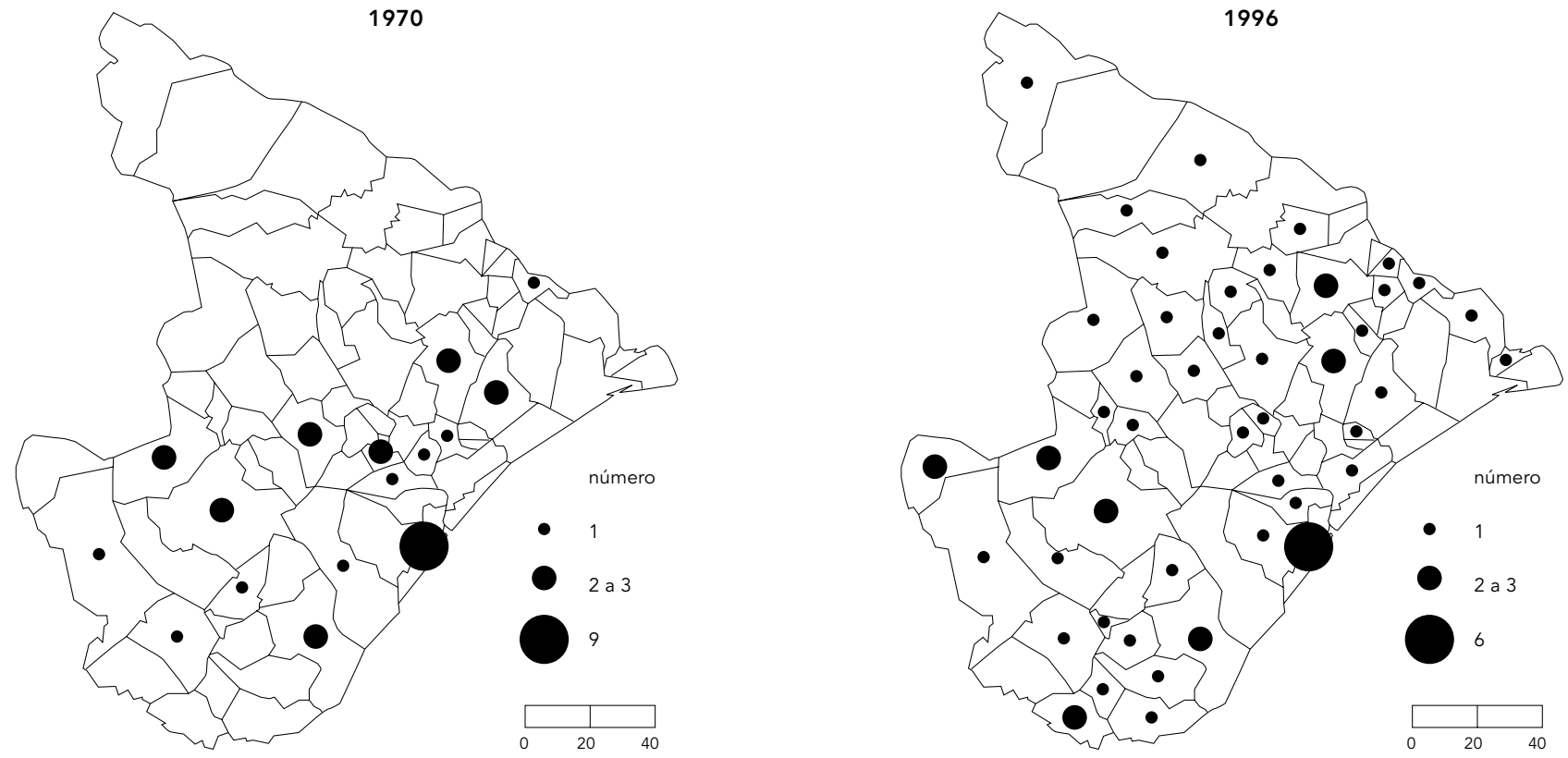

Figura 4

Maternidades. Sergipe, Brasil, 1970 e 1996.
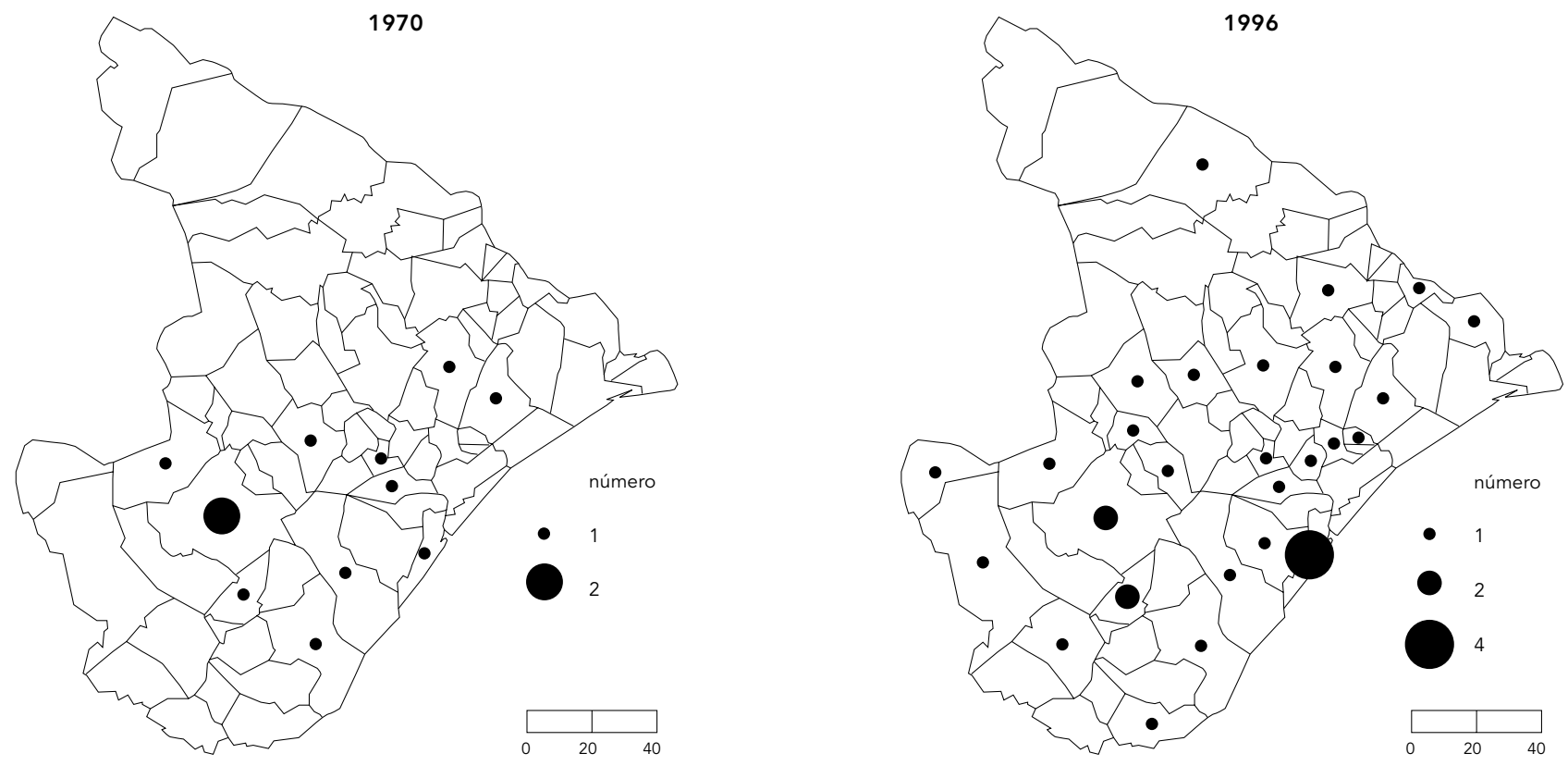
pios, penetrando pela Bahia. Avalia-se que a menor incidência de partos dos municípios mais distantes se dá em função da presença de maternidades em centros regionais, como Nossa Senhora da Glória, Itabaiana, Estância, Lagarto e Propriá. Mesmo com a presença de hospitais e maternidades nos centros regionais, a migração continua intensa, favorecida pela melhoria das estradas e do sistema de transportes, além do apoio de políticos e das prefeituras municipais.

É sabido que gestações complicadas, com risco para a gestante ou para o feto, atendidas em hospitais sem recursos devem transferir o parto para um hospital com melhores condições. A transferência, quando necessária, deveria ocorrer de preferência para um centro regional, como é o caso de Itabaiana ou Estância, por exemplo, diminuindo o tempo de transporte e, conseqüentemente, as complicações devidas ao trajeto e reduzindo a sobrecarga de partos em Aracaju. Itabaiana faz parte do DRS II onde, apesar de ter havido importante incremento na variação relativa de partos desse DRS nos períodos 1970-1976 e 1976-1986, houve variação negativa de $-61,17 \%$ em 1986-1996. O DRS I, cujo centro regional é Estância também apresentou variação negativa de $-22,22 \%$ no mesmo período. Assim, infere-se que tem havi- do melhoria na qualidade dos serviços dessas cidades.

Campos \& Carvalho (2000) mapearam o fluxo das gestantes no Município do Rio de Janeiro e verificaram que, em geral, esses fluxos apontam no sentido das regiões mais ricas da cidade, ligando as parturientes que residem em áreas de baixa renda a locais onde se encontram maiores e melhores ofertas de serviços de saúde. Isso é o que parece estar ocorrendo em Sergipe no período estudado. De fato, tem-se uma migração para o centro que dispõe de melhores condições técnicas. No início, essa migração era feita por pessoas de melhor poder aquisitivo. Posteriormente, houve uma disseminação do processo que hoje envolve todas as categorias sociais, também em função da ampliação do SUS.

Os dados retratam um movimento de procura por melhores condições de atenção à saúde perinatal em Sergipe, no período de 1970 a 1996, ocorrido sem um planejamento explícito relatado, podendo-se identificar razões de cunho político ideológico nocivas à população. Trabalhos dessa natureza tornam-se valiosos para o entendimento de fenômenos de saúde de uma área, permitindo melhorias de planejamento de ações de saúde.

\section{Referências}

BETTIOL, H.; BARBIERI, M. A.; GOMES, U. A.; ANDREA, M.; GOLDANI, M. Z.; RIBEIRO, E. R. O., 1998. Saúde perinatal: Metodologia e características da população estudada. Revista de Saúde Pública, 32:18-28.

BUSS, P. M., 1993. Assistência hospitalar no Brasil (1984-1991): Uma análise preliminar baseada no Sistema de Informação Hospitalar do SUS. Informe Epidemiológico do SUS, mar/abr:5-17.

CAMPOS, T. P. \& CARVALHO, M. S., 2000. Assistência ao parto no Município do Rio de Janeiro: Perfil das maternidades e o acesso da clientela. Cadernos de Saúde Pública, 16:411-420.

CAMPOS, T. P.; CARVALHO, M. S. \& BARCELLOS, C. C., 2000. Mortalidade infantil no Rio de Janeiro, Brasil: Áreas de risco e trajetória dos pacientes até os serviços de saúde. Revista Panamericana de Salud Publica, 8:164-171.

CONDESE (Conselho de Desenvolvimento de Sergipe), 1971. Anuário Estatístico de Sergipe. Aracaju: CONDESE. 
DER-SE (Departamento de Estradas de Rodagens de Sergipe), 1974. Sergipe Rodoviário. Salvador: Artes Gráficas.

DER-SE (Departamento de Estradas de Rodagens de Sergipe), 1979. Sergipe Rodoviário 3. Aracaju: J. Andrade.

DER-SE (Departamento de Estradas de Rodagens de Sergipe), 1982. Sergipe Rodoviário 4. Aracaju: J. Andrade.

DER-SE (Departamento de Estradas de Rodagens de Sergipe), 1987. Sergipe Rodoviário 7. Aracaju: J. Andrade.

DETRAN-SE (Departamento de Trânsito de Sergipe), 2001. Dados Cadastrais de Ambulâncias. Aracaju: DETRAN-SE.

FRANÇA, S. V. A. \& TORRES, J. S., 1998. Aprendendo Atlas GIS. Aracaju: Núcleo de Pós-Graduação em Geografia, Universidade Federal de Sergipe. (mimeo.)

FRANÇA, V. L. A., 1999. Aracaju: Estado e Metropolização. Aracaju: Universidade Federal de Sergipe/ Fundação Oviêdo Teixeira.

GOMES, J. O. \& SANTO, A. H., 1997. Mortalidade infantil em município da região Centro-Oeste Paulista, Brasil, 1990 a 1992. Revista de Saúde Pública, 31:330-341.

GOUVÊA, C. S. D.; TRAVASSOS, C. \& FERNANDES, C., 1997. Produção de serviços e qualidade da assistência hospitalar no Estado do Rio de Janeiro, Brasil - 1992 a 1995. Revista de Saúde Pública, 31:601-617.

IBGE (Fundação Instituto Brasileiro de Geografia e Estatística), 1970. Censo Demográfico, 1970. Rio de Janeiro: IBGE.

IBGE (Fundação Instituto Brasileiro de Geografia e Estatística), 1991. Censo Demográfico, 1991. Rio de Janeiro: IBGE.

LEAL, M. C. \& SZWARCWALD, C. L., 1996. Evolução da mortalidade neonatal no Estado do Rio de Janeiro, Brasil, de 1979 a 1993. 1 - Análise por grupo etário segundo região de residência. Revista de Saúde Pública, 30:403-412.
MENDONZA-SASSI, R. \& BERIA, J. U., 2001. Utilización de los servicios de salud: Una revisión sistemática sobre los factores relacionados. Cadernos de Saúde Pública, 17:819-832.

MORAIS NETO, O. L.; BARROS, M. B. A.; MARTELLI, C. M. T.; SILVA, S. A.; CAVENAGHI, S. M. \& SIQUEIRA Jr., J. B., 2001. Diferenças no padrão de ocorrência da mortalidade neonatal e pós-neonatal no Município de Goiânia, Brasil, 1992-1996: Análise espacial para identificação das áreas de risco. Cadernos de Saúde Pública, 17:1241-1250.

RIBEIRO, V. S. \& SILVA, A. A. M., 2000. Tendências da mortalidade neonatal em São Luís, Maranhão, Brasil, de 1979 a 1996. Cadernos de Saúde Pública, 16:429-438.

SECRETARIA DE ESTADO DA SAÚDE DE SERGIPE, 1987. Modelo Assistencial de Sergipe. Aracaju: Sistema Unificado e Descentralizado de Saúde.

SEPLAN (Secretaria de Estado de Planejamento), 1987. Anuário Estatístico de Sergipe. Aracaju: SEPLAN.

SEPLANTEC (Secretaria de Estado do Planejamento e da Ciência e Tecnologia), 1997. Anuário Estatístico de Sergipe. Aracaju: SEPLANTEC.

SHIMAKURA, S. E.; CARVALHO, M. S.; AERTS, D. R. C \& FLORES, R., 2001. Distribuição espacial do risco: Modelagem da mortalidade infantil em Porto Alegre, Rio Grande do Sul, Brasil. Cadernos de Saúde Pública, 17:1251-1261.

SILVA, N. N.; PEDROSO, G. C.; PUCCINI, R. F. \& FURLANI, W. J., 2000. Desigualdades sociais e uso de serviços de saúde: Evidências de análise estratificada. Revista de Saúde Pública, 34:44-49.

TOWNSEND, P. \& DAVIDSON, N. 1982. Inequalities in Health: The Black Report. London: Penguin Books.

VICTORA, C. G.; GRASSI, P. R. \& SCHMIDT, A. M., 1994. Situação de saúde da criança em área da região sul do Brasil, 1980 - 1992: Tendências temporais e distribuição espacial. Revista de Saúde Pública, 28:423-432.

Recebido em 15 de agosto de 2001

Versão final reapresentada em 6 de maio de 2002

Aprovado em 19 de agosto de 2002 